\title{
ON THE INFLUENCE OF MARANGONI CONVECTION ON THE STABILITY OF LIQUID BRIDGE INTERFACES
}

\author{
I.E. Parra, J. M. Perales, and J. Meseguer \\ IDR/UPM. E.T.S.I. Aeronáuticos, Universidad Politécnica de Madrid, E-28040 Madrid, Spain
}

\begin{abstract}
This paper analyses the influence of the Marangoni flow on the stability of almost cylindrical liquid bridges by using bifurcation techniques. An analytical relation between the different parameters is found that allows the prediction of the variation of the maximum stable length. This variation although is qualitatively "larger" than that due to symmetric effects (f.i. volume variation, solid body rotation), can somehow be compensated with the other effects. By choosing adequately f.j. the disk diameter difference, the shortening in stability due to Marangoni convection can be almost completely cancelled.
\end{abstract}

\section{INTRODUCTION AND GOVERNING EQUATIONS}

It is well known (see for example Meseguer et al. (1999) for a recent review) that the classical Rayleigh limit (a cylindrical shaped liquid bridge loses its stability when its length is larger than its perimeter) is modified (and shortened) by the influence of many parameters (unequal diameter supports, liquid volume not precisely that of the cylinder, axial or lateral accelerations, solid body rotation, ...). It is also well known that, in some real applications of this idealized model (f.i. the floating zone crystal growth technique), there is a temperature gradient that, due to the variation of surface tension with temperature, drives a flow (the Marangoni convection) in the liquid bulk. The Marangoni convection that appears is usually modelled by the so called half zone model where temperatures of the circular supports are kept constant but different. In this case, the forced flow is antisymmetric with respect to the mid plane of the liquid bridge. Thus, one must expect for small Marangoni flows a variation in the Rayleigh limit qualitatively similar to that due to other antisymmetric effects (axial acceleration, disk diameter inequality).

Let us assume an axisymmetric mass of liquid of volume $\bar{V}$ held by surface tension forces between two solid coaxial disks of radii $R_{1}$ and $R_{2}$, respectively, whose centres are placed a distance $L$ apart and let $g$ be the axial acceleration acting on the liquid mass, which is assumed to be constant. The liquid is assumed to be a Newtonian fluid with temperature independent thermodynamic and transport properties (density, $\rho_{l}$, viscosity, $\mu_{i}$, thermal conductivity, $k_{k}$, specific heat, $c_{r}$, and convective coefficient, $h_{i}$ ), and with a surface tension, $\bar{\sigma}$, varying linearly with temperature, $\bar{T}: \bar{\sigma}=\bar{\sigma}_{0}+(\mathrm{d} \bar{\sigma} / \mathrm{d} \bar{T})\left(\bar{T}-\bar{T}_{0}\right)$, where $\bar{\sigma}_{0}$ is the value of the surface tension at the temperature of reference $\bar{T}_{0}$, and the variation of the surface tension with temperature is assumed to be initially negative .

Since the temperature is assumed to deviate only slightly from its reference value, in a first attempt the liquid density can be assumed to be constant. Therefore, in dimensionless variables, the conservation equations for the steady flow are: $\nabla \cdot \mathbf{V}=0$ (mass conservation), $\nabla P=\mathbf{B k}+\operatorname{Cr} \Delta \mathbf{V}-\operatorname{CrRe}(\mathbf{V} \cdot \nabla) \mathbf{V}$ (momentum conservation) and $\Delta T=\operatorname{Re} \operatorname{Pr} V \cdot \nabla T$ (energy conservation) where $\mathbf{V}$ is the non-dimensional velocity vector, whose components are $U, V$, and $W . P$ and $T$ stands for the dimensionless pressure and temperature, and the Bond number, $\mathrm{B}$, crispation number, $\mathrm{Cr}$, Reynolds number, Re, and Prandtl number, $\mathrm{Pr}$, are defined in the following. In dimensionless form the variation of surface rension with temperature reads $\sigma=\tilde{\sigma} / \bar{\sigma}_{0}=1-\mathrm{Cr} T$. In the above equations all lengths have been made dimensionless with the mean radius $R=\left(R_{1}+R_{2}\right) / 2$, and velocities have been made dimensionless with the characteristic velocity $U_{0}$, defined as $U_{0}=-(\mathrm{d} \bar{\sigma} / \mathrm{d} \bar{T}) \bar{T}_{0} / \mu_{i}$. Pressure $P$ has been made dimensionless with the characteristic pressure $\bar{\sigma}_{0} / R$, and the dimensionless temperature $T$ has been defined as $T=\left(\bar{T}-\bar{T}_{0}\right) / \bar{T}_{0}$. 
Dimensionless parameters defining the geometry are the slenderness, $\Lambda=L /(2 R)$ and a dimensionless parameter measuring disk radii difference $h=\left(R_{1}-R_{2}\right) /\left(R_{1}+R_{2}\right)$, where $R_{2}$ is the radius of the upper disk and $R_{2}$ that of the lower one. Other parameters are the dimensionless volume, defined as the ratio of the actual volume $\bar{V}$ to the volume of a cylinder of the same length $L$ and diameter $2 R: V^{*}=\bar{V} / \pi R^{2} L$, the Bond number, $\mathrm{B}=\Delta \rho_{I} g R^{2} / \vec{\sigma}_{0}$, where $\Delta \rho_{l}=\rho_{l}-\rho_{m}$ is the difference between the density of the liquid forming the liquid bridge, $\rho_{l}$ and the density of the surrounding medium, $\rho_{m}$, which is assumed to be negligible when compared with $\rho_{l}\left(\rho_{i}>>\rho_{i n}\right)$. Other dimensionless parameters involved in the problem formulation are the crispation number, $\mathrm{Cr}=-(\mathrm{d} \bar{\sigma} / \mathrm{d} \bar{T}) \bar{T}_{0} / \bar{\sigma}_{0}$, the Reynolds number, $\operatorname{Re}=\rho_{l} \bar{U}_{0} R / \mu_{l}$, the Prandtl number, $\operatorname{Pr}=\mu_{l} c_{p} / k_{l}$, and the Biot number, $\mathrm{Bi}=h_{l} R / k_{l}$.

Dimensionless kinematic and thermal boundary conditions at the solid disks are, respectively $V( \pm \Lambda, r)=0$, $T( \pm \Lambda, r)= \pm \alpha / \mathrm{Cr}$ where $\alpha=\mathrm{Cr} \Delta \bar{T}_{0} / \bar{T}_{0}$. Boundary conditions at the disks are completed with the disk anchoring condition $F( \pm \Lambda)=l \pm h$, where $r=F(z)$ represents the liquid bridge interface. At the liquid bridge interface dynamic boundary conditions state the equilibrium at the free surface both in the normal direction to the interface and in the tangential direction. Such boundary conditions read in dimensionless form respectively:

$$
\begin{aligned}
& P+\sigma\left[F_{z z}-\left(1+F_{z}^{2}\right) / F\right]\left[1+F_{z}^{2}\right]^{-3 / 2}=-2 \mathrm{Cr}\left[U_{r}-F_{z}\left(U_{z}+W_{r}\right)+F_{z}^{2} W_{z}\right]\left[1+F_{z}^{2}\right]^{-1}, \\
& F_{z} T_{r}+T_{z}=-\left[2 F_{z} U_{r}+\left(1-F_{z}^{2}\right)\left(U_{z}+W_{r}\right)-2 F_{z} W_{z}\right]\left[1+F_{z}^{2}\right]^{-1 / 2} .
\end{aligned}
$$

In addition, a kinematic boundary condition at the liquid bridge interface results by expressing that the liquid interface, $r=F(z)$, is a fluid surface: $U-W F_{z}=0$, and the thermal boundary condition at the interface is deduced from the surface balance equation for the energy:

$$
\left[T_{r}-F_{z} T_{z}\right]\left[1+F_{z}^{2}\right]^{-1 / 2}=-\mathrm{Bi}\left(T-T_{\mathrm{ext}}\right),
$$

$T_{\mathrm{ex}}$ being the dimensionless temperature of the surrounding medium. Finally, to determine pressure level, the liquid volume preservation condition is needed:

$$
\pi \int_{-\Lambda}^{\Lambda} F^{2} \mathrm{~d} z=2 \pi \Lambda V^{*}
$$

\section{ASYMPTOTIC EXPANSIONS AND BIFURCATION TO UNSTABLE EQUILIBRIUM SHAPES}

To analyse the asymptotic stability limits of axisymmetric long liquid bridges, in the following only the limiting case of zero Reynolds number and zero Biot number is considered. Under these restrictive circumstances $(\mathrm{Re}=\mathrm{Bi}=0)$ and the additional limiting hypothesis of axisymmetric configuration (the velocity vector has only two components, $U$ and $W$, the introduction of the stream function for axisymmetric flow, $\chi(U=\partial(r \chi) / \partial z$. $W=-(1 / r) \partial(r \chi) / \partial r)$ automatically fulfils continuity equation; in such case the energy equation reduces to the Laplace equation for the temperature whereas the momentum equation reduces to the biharmonic differential equation for $\chi \cdot \Delta^{2} \chi=0$ to be integrated with the following boundary conditions at the disks:

$$
\left.\frac{\partial \chi}{\partial z}\right|_{z= \pm \Lambda}=\left.\frac{\partial \chi}{\partial r}\right|_{z= \pm \Lambda}=\left.\chi\right|_{z= \pm \Lambda}=0, T( \pm \Lambda, r)= \pm \alpha / \mathrm{Cr}, F( \pm \Lambda)=1 \pm h
$$

At the interface, $r=F(z)$, boundary conditions are given by Eqs. (1)-(2), plus the kinematic boundary condition and the thermal boundary condition ( $\mathrm{Eq} .3$ ), the latter with $\mathrm{Bi}=0$, and additionally, the volume preservation condition (Eq. 4) must hold.

Under the additional constraints of isothermal liquid bridge $(\alpha=0)$, zero axial acceleration $(B=0)$, disks of equal radii $(h=0)$ and cylindrical volume of liquid $\left(V^{*}=1\right)$, the above formulation for the axisymmetric problem, has the trivial equilibrium solution $T=0, v=0, P=I$ and $F=1$ for any value of the slenderness $A$. However, it is well-known that this liquid bridge configuration $(F=1)$ becomes unstable when the distance between the supporting disks equals the length of the disk circumference, the so-called Rayleigh limit $\Lambda=\pi$. Such stability limit is obtained after the introduction of the following expansions in the problem formulation $F(z)=1+\varepsilon \hat{f}(z)+\ldots, P(z, r)=1+\varepsilon \hat{p}(z, r)+\ldots, T(z, r)=\varepsilon \hat{t}(z, r)+\ldots, \quad \mathbf{V}(z, r)=\varepsilon \hat{v}(z, r)+\ldots$, where $\varepsilon$ stands for the magnitude of the deformation of the interface, which allows one to calculate the first order terms of the above expansion after neglecting $O\left(\varepsilon^{2}\right)$ terms. Non-trivial (different from zero) solutions of the linear problem accur only for a discrete number of values of $\Lambda$. The smallest value of $\Lambda$ for which a non-trivial (bifurcation to non-cylindrical 
equilibrium shapes) appears is for $\Lambda=\pi$, as already said. Here the transition from stable to unstable equilibrium shapes occurs (all other bifurcation points are irrelevant as they cannot be attained because the liquid bridge will break for $\Lambda>\pi$ ). Therefore, the only relevant instability appears at $\Lambda=\pi$ and, within this approximation, the departure from the cylinder of the unstable equilibrium shapes are defined by $\hat{f}(z)=\sin (\pi z / \Lambda), \hat{p}(z, r)=0$, $\hat{i}(z, r)=0, \hat{\mathbf{v}}(z, r)=0$, which is the solution that must be perturbed to calculate the variation of the maximum stable stenderness. From this point on, the procedure used to obtain the variation of the maximum stable slenderness due to the different effects under consideration is similar to that described by Perales (1987) and Meseguer et al. (1995). First, a new variable, $x=\pi z / \Lambda$, which normalizes boundary conditions, is introduced along with the parameter $\lambda=1-\Lambda / \pi$, and terms of higher order than those appearing in the linear problem are retained. Let $f(x), p(x, r), t(x, r)$ and $\mathbf{v}(x, r)$ represent these higher order terms in the expansions of the interface shape, the pressure, the temperature and the velocity, respectively. The new expansions for $F, P, T$ and $\mathrm{V}$ are then $F(z)=1+\varepsilon \sin x+f(x), P=1+p(x, r), T(x, r)=t(x, r), \quad \mathbf{V}(x, r)=\mathbf{v}(x, r)$. Note that this new formulation requires an additional condition to uniquely define the parameter $\varepsilon$, that is,

$$
\int_{-\pi}^{\pi} f \sin x \mathrm{~d} x=0
$$

The above defined problem allows us to calculate $f(x), p(x, r), t(x, r)$ and $v(x, r)$ in terms of the small parameters $\varepsilon, \lambda, v, h, \mathrm{~B}$ and $\alpha$, where $v=V^{*}-1$ is the small parameter that accounts for the difference between actual liquid bridge volume and the cylindrical one. Although a direct solution could be attempted for small values of the parameters involved, it requires that the relative orders of magnitude of the parameters be anticipated. Instead, a systematic approach based on the bifurcation equation will be used following Vega and Perales (1983). Thus, instead of the boundary condition (1) the new equation to be solved is

$$
P+\sigma\left[F_{z z}-\left(1+F_{z}^{2}\right) / F\right]\left[1+F_{z}^{2}\right]^{-3 / 2}+2 \mathrm{Cr}\left[U_{r}-F_{z}\left(U_{z}+W_{r}\right)+F_{z}^{2} W_{z}\right]\left[1+F_{z}^{2}\right]^{-1}+\phi \sin x=0 .
$$

From the Implicit Function Theorem (Chow and Hale, 1982) it can be demonstrated that now, at least in a neighborhood of $\varepsilon=\lambda=v=h=\mathrm{B}=\alpha=0$ the new problem formulation uniquely define $f, p, t, \mathrm{v}$ and $\phi$ in terms of a power series in the parameters. Such solutions will correspond to the solution of original set of equations if and only if the parameters involved satisfy the bifurcation equation $\phi(\varepsilon, \lambda, v, h, \mathrm{~B}, \alpha)=0$.

Most of the first, second and third order problems (all which do not contain $\alpha$ in its coefficient) leads to the trivial solution for the temperature and velocities, $t=0, \mathbf{v}=\mathbf{0}$. The solution of these problems is then identical to those reported by Meseguer et al. (1995). As can be found there the relevant coefficients are: $\phi_{h}=-2 / \pi, \phi_{B}=2$, $\phi_{\varepsilon \lambda}=2, \phi_{\varepsilon i}=\mathrm{I}, \phi_{c \varepsilon t}=-3 / 2$, (additional details can be obtained upon request to the authors). The only new problem that requires a detailed analysis is the $\alpha$-order problem because of the non trivial solution of the thermal problem

$$
\frac{\partial^{2} t_{\alpha}}{\partial r^{2}}+\frac{1}{r} \frac{\partial t_{\alpha}}{\partial r}+\frac{\partial^{2} t_{\alpha}}{\partial x^{2}}=0, t_{\alpha}( \pm \pi, r)= \pm 1,\left.\frac{\partial t_{\alpha}}{\partial r}\right|_{r=1}=0,
$$

which is $t_{\alpha}(x, r)=x / \pi$. In this case, the problem to be solved for the stream function $\chi_{\alpha}$ is:

$$
\left(\frac{\partial^{2}}{\partial r^{2}}+\frac{1}{r} \frac{\partial}{\partial r}+\frac{\partial^{2}}{\partial x^{2}}-\frac{1}{r^{2}}\right)^{2} \chi_{\alpha}=0,\left.\left(\frac{\partial^{2} \chi_{\alpha}}{\partial r^{2}}+\frac{1}{r} \frac{\partial \chi_{\alpha}}{\partial r}-\frac{\chi_{\alpha}}{r^{2}}\right)\right|_{r=1}=\frac{1}{\pi},\left.\frac{\partial \chi_{\alpha}}{\partial x}\right|_{r=1}=0,\left.\chi_{\alpha}\right|_{x= \pm \pi}=\left.\frac{\partial \chi_{\alpha}}{\partial x}\right|_{x= \pm \pi}=0
$$

This problem was already solved by Da Riva and Alvarez Pereira (1982), yielding the following expressions for the velocity components

$$
u_{\alpha}(x, r)=-\frac{1}{\pi} \sum_{-\infty}^{\infty} \frac{1}{S_{n}^{2} \cos ^{4}\left(S_{n} \pi\right)} \frac{\mathrm{I}_{1}\left(S_{n} r\right)}{\mathrm{I}_{1}\left(S_{n}\right)} \frac{\mathrm{d} \bar{\phi}_{1}^{(n)}(x)}{\mathrm{d} x} \quad, \quad w_{\alpha}(x, r)=\frac{1}{\pi} \sum_{-\infty}^{\infty} \frac{1}{S_{n} \cos ^{4}\left(S_{n} \pi\right)} \frac{\mathrm{I}_{0}\left(S_{n} r\right)}{\mathrm{I}_{1}\left(S_{n}\right)} \tilde{\phi}_{1}^{(n)}(x),
$$

where $\tilde{\phi}_{1}^{(n)}(x)=\pi S_{n} \sin \left(S_{n} \pi\right) \cos \left(S_{n} x\right)-S_{n} x \cos \left(S_{n} \pi\right) \sin \left(S_{n} x\right)$ are the first even Papkovich-Fadle functions and the eigenvalues $S_{n}$ are the first-quadrant complex roots of $\sin \left(2 \pi S_{n}\right)+2 \pi S_{n}=0 . I_{0}$ and $l_{1}$ are the modified Bessel functions of order zero and order one, respectively. To calculate the pressure field the equations to be solved are:

$$
\frac{\partial p_{\alpha}}{\partial r}=\frac{\partial^{2} u_{\alpha}}{\partial r^{2}}+\frac{1}{r} \frac{\partial u_{\alpha}}{\partial r}+\frac{\partial^{2} u_{\alpha}}{\partial x^{2}}-\frac{u_{\alpha}}{r^{2}} \quad, \quad \frac{\partial p_{\alpha}}{\partial x}=\frac{\partial^{2} w_{\alpha}}{\partial r^{2}}+\frac{1}{r} \frac{\partial w_{\alpha}}{\partial r}+\frac{\partial^{2} w_{\alpha}}{\partial x^{2}}
$$

which give the following result for the pressure: 


$$
p_{\alpha}(x, r)=\tilde{p}_{\alpha}-\frac{2}{\pi} \sum_{-\infty}^{\infty} \frac{1}{\cos ^{3}\left(S_{n} \pi\right)} \frac{\mathrm{I}_{0}\left(S_{n} r\right)}{\mathrm{I}_{1}\left(S_{n}\right)} \sin \left(S_{n} x\right) .
$$

The function $g_{\alpha}$, the constant pressure of reference $p_{\alpha}$ as well as the coefficient of the bifurcation equation $\phi_{\alpha}$ are obtained after integration of the $\alpha$-order problem (for the liquid interface shape), which now reads:

$$
\frac{\partial^{2} g_{a}}{\partial x^{2}}+g_{\alpha}+p_{\alpha}+\frac{x}{\pi}+\frac{2}{\pi} \sum_{-\infty}^{\infty} \frac{S_{n}}{\cos ^{4}\left(S_{n} \pi\right)} \frac{\mathrm{I}_{0}\left(S_{n}\right)}{I_{1}\left(S_{n}\right)}\left[\pi \sin \left(S_{n} \pi\right) \sin \left(S_{n} x\right)+x \cos \left(S_{n} \pi\right) \cos \left(S_{n} x\right)\right]+\phi_{\alpha} \sin x=0,
$$

with the boundary conditions $g_{\alpha}( \pm \pi)=0, \int_{-\pi}^{\pi} g_{\alpha} \mathrm{d} x=0, \int_{-\pi}^{\pi} g_{\alpha} \sin x \mathrm{~d} x=0$.

Note that two new different terms appear in Eq. (13). One of them (the $x / \pi$ term) is due to surface tension variation along the interface whereas the other (the summation term) is due to the pressure change due to the inner flow. The resulting expression for $\phi_{a}$ is:

$$
\phi_{\alpha}=-\frac{2}{\pi}+\frac{4}{\pi} \sum_{-\infty}^{\infty} \frac{S_{n}\left(3 S_{n}^{2}-1\right)}{\left(S_{n}^{2}-1\right)^{2} \cos ^{4}\left(S_{n} \pi\right)} \frac{\mathrm{I}_{0}\left(S_{n}\right)}{\mathrm{I}_{1}\left(S_{n}\right)}=2.1589 .
$$

Note that a difference in the temperatures of the disks gives an interface deformation which is non-symmetric with respect to the mid plane. Thus, the bifurcation equation is now $-(2 / \pi) h+2 \mathrm{~B}+\phi_{\alpha} \alpha+2 \varepsilon \lambda+\varepsilon v-(3 / 2) \varepsilon^{3}+\ldots=0$, where only the leading terms for each parameter have been written and (non-zero) terms in $\lambda h, \lambda \mathrm{B}, v h$, and $v \mathrm{~B}$ have been omitted. The stability limit is then $\lambda=\lambda_{\min }$, or $\Lambda_{\max }=\pi\left(1-\lambda_{\text {min }}\right)$. Taking $\mathrm{d} \lambda / \mathrm{d} \varepsilon=0$, to leading order we find:

$$
\Lambda_{\max }=\pi\left\{1-\left(\frac{3}{2}\right)^{4 / 3}\left[\mathrm{~B}-\frac{h}{\pi}+\frac{1}{2} \phi_{\alpha} \alpha\right]^{2 / 3}+\frac{1}{2} v\right\} .
$$

The quantitative influence of the different parameters is determined by the exponents of each group of terms in Eq. (15) and can be understood by considering the effect of the imposed perturbation on the necking of the liquid column. The expression for both stable and unstable equilibrium interface shapes is of the form $f(x)=1+\varepsilon \sin x$. When the instability develops, the liquid bridge interface bulges in one half of the liquid column and necks in the other (the instability is antisymmetric with respect to the mid-plane parallel to the disks). According to this behavior, any perturbation leading to an antisymmetric deformation of the interface will decrease the stability limit. Note that the reduction of the maximum stable slenderness is proportional to the two-thirds power of the perturbation. Perturbations that cause a symmetric deformation (with respect to the mid-plane) of the interface, such as those due to a reduction in volume, may also reduce the stability limit. However, these kinds of perturbation are less critical and now the reduction in $\Lambda_{\max }$ is linear in the volume, $v$. Note that, for certain combinations of the parameters, namely when $\mathrm{B}-h / \pi+\phi_{\alpha} \alpha / 2=0$, the change in the stability limit due to the Marangoni convection is exactly compensated by that due to Bond number and disk unequality.

\section{ACKNOWLEDGEMENTS}

This work has been supported by the Spanish Ministerio de Ciencia y Tecnologia (MCYT).

\section{REFERENCES}

Chow, N.S. and Hale, J.K., Methods of bifurcation theory, Springer-Verlag, Berlin, 1982.

Da Riva, I. and Alvarez Pereira, E., A regular perturbation approach to surface tension driven flows, Acta Astron. 9, $217,1982$.

Meseguer, J., Bezdenejnykh. N.A., Perales, J.M. and Rodríguez de Francisco, P., Theoretical and experimental analysis of stability limits of non-axisymmetric liquid bridges under microgravity conditions, Microgravity Sci. Technol. 8, 2-9, 1995.

Meseguer, J., Perales, J.M., Martínez, I, Bezdenejnykh, N.A. and Sanz, A., Hydrostatic instabilities in floating zone crystal growth process, Current Topics in Cryst. Growth Res. 5, 27-42, 1999.

Perales, J.M., Non-axisymmetric effects on long liquid bridges, Acta Astron. 15, 561-565, 1987.

Vega, J.M. and Perales, J.M., Almost Cylindrical Isorotating Liquid Bridges for small Bond Numbers, in Materials Sciences under Microgravity, ESA SP-191, pp 247-252, 1983. 